\title{
Under Pressure: Financial Effect of the Hospital-Acquired Conditions Initiative-A Statewide Analysis of Pressure Ulcer Development and Payment
}

\author{
Jennifer Meddings, MD, MSc, "Heidi Reichert, MA, * Mary A. M. Rogers, PhD, MS, * \\ Timothy P. Hofer, MD, MS, *广 Laurence F. McMahon, Jr, MD, MPH, *t and Kyle L. Grazier, PhD
}

OBJECTIVES: To assess the financial effect of the 2008 Hospital-Acquired Conditions Initiative (HACI) pressure ulcer payment changes on Medicare, other payers, and hospitals.

DESIGN: Retrospective before-and-after study of all-payer statewide administrative data for more than 2.4 million annual adult discharges in 2007 and 2009 using the Healthcare Cost and Utilization Project State Inpatient Datasets for California. How often and by how much the 2008 payment changes for pressure ulcers affected hospital payment was assessed.

SETTING: Nonfederal acute care California hospitals $(\mathrm{N}=311)$.

PARTICIPANTS: Adults discharged from acute-care hospitals.

MEASUREMENTS: Pressure ulcer rates and hospital payment changes.

RESULTS: Hospital-acquired pressure ulcer rates were low in $2007(0.28 \%)$ and 2009 (0.27\%); present-onadmission pressure ulcer rates increased from $2.3 \%$ in 2007 to $3.0 \%$ in 2009 . According to clinical stage of pressure ulcer (available in 2009), hospital-acquired Stage III and IV ulcers occurred in 603 discharges (0.02\%); 60,244 discharges $(2.42 \%)$ contained other pressure ulcer diagnoses. Payment removal for Stage III and IV hospitalacquired ulcers reduced payment in $75(0.003 \%)$ discharges, for a statewide payment decrease of $\$ 310,444$ $(0.001 \%)$ for all payers and \$199,238 (0.001\%) for Medicare. For all other pressure ulcers, the Hospital-Acquired Conditions Initiative reduced hospital payment in 20,246 $(0.81 \%)$ cases (including 18,953 cases with present-onadmission ulcers), reducing statewide payment by

From the *Division of General Medicine, Department of Internal Medicine, University of Michigan Medical School; ${ }^{\dagger}$ Veterans Affairs Ann Arbor Healthcare System; and "University of Michigan School of Public Health, Ann Arbor, Michigan.

Address correspondence to Jennifer Meddings, 2800 Plymouth Road, Building 16, Room 430W, Ann Arbor, MI 48109. E-mail:

meddings@umich.edu

DOI: $10.1111 /$ jgs. 13475
$\$ 62,538,586(0.21 \%)$ for all payers and $\$ 47,237,984$ $(0.32 \%)$ for Medicare.

CONCLUSION: The total financial effect of the 2008 payment changes for pressure ulcers was negligible. Most payment decreases occurred by removal of comorbidity payments for present-on-admission pressure ulcers other than Stages III and IV. The removal of payment for hospital-acquired Stage III and IV ulcers by implementation of the HACI policy was $1 / 200$ th that of the removal of payment for other types of pressure ulcers that occurred in implementation of the Hospital-Acquired Conditions Initiative. J Am Geriatr Soc 63:1407-1412, 2015.

Key words: hospital-acquired conditions; pressure ulcer; payment; Medicare

$\mathbf{P}$ ressure ulcers (bed sores), are skin or tissue injuries over bony prominences caused by pressure and/or shear that range in severity from Stage I (nonblanchable erythematous intact skin) to Stage IV (with full-thickness tissue loss exposing bone, tendon, or muscle). ${ }^{1}$ Hospital-acquired pressure ulcers are common ( 1 million to 2.5 million annually in the United States $\left.{ }^{2,3}\right)$, painful, ${ }^{4}$ expensive, ${ }^{2,3,5,6}$ often preventable, ${ }^{7}$ and potentially life threatening. ${ }^{3}$ Cost estimates to heal a single pressure ulcer vary according to severity and population studied from hundreds of dollars ${ }^{2}$ for Stage $\mathrm{I}$ and II ulcers tod $\$ 5,000$ to $\$ 151,700^{3,5,6}$ for moreadvanced ulcers of Stage III or IV.

Value-based purchasing programs ${ }^{8-12}$ are used to motivate hospitals to prevent complications such as pressure ulcers using payment changes. For example, the October 2008 Hospital-Acquired Conditions Initiative $(\mathrm{HACI})^{10,11,13,14}$ eliminated extra Medicare payments for treating certain complications, including pressure ulcers. As detailed in official Centers for Medicare and Medicaid Services (CMS) information ${ }^{9,10,14}$ in press releases and on-line material prepared for Medicare providers about the HACI, advanced-stage pressure ulcers (Stage III or IV) can no longer generate extra payment as a comorbidity when a 
hospital-acquired diagnosis. Somewhat inexplicably (described in implementation details of the HACI in the Federal Register ${ }^{13}$ ), earlier-stage (Stage I and II) pressure ulcers and unstageable and stage-not-specified ulcers also no longer generate extra payment as comorbidities whether hospital-acquired or present-on-admission diagnoses. Payment removal for these present-on-admission pressure ulcers appears to be a pure cost-cutting decision because hospitals cannot influence their development. In this study, two analyses assessing the effect of the 2008 HACI were performed using the administrative data upon which this policy is implemented. First, the overall changes in pressure ulcer rates categorized as present-on-admission versus hospital-acquired diagnoses were assessed using 2007 (pre-policy) data and 2009 (postpolicy) data, without respect to pressure ulcer stage because pressure ulcer stage codes were not available in 2007. Next, reductions in hospital payment were calculated that occurred in 2009 resulting from changes in pressure ulcer payment according to pressure ulcer stage and status on admission as required after implementation of the 2008 HACI.

\section{METHODS}

\section{Study Design and Data Sources}

Using administrative discharge data from the Healthcare Cost and Utilization Project State Inpatient Datasets ${ }^{15}$ for California in 2007 and 2009, Medicare Severity Diagnosis Related Groups (MS-DRGs) Grouper software, and hospital payment files from the CMS, a retrospective beforeand-after study was conducted to assess how often and by how much hospital payments decreased as a result of the 2008 pressure ulcer payment changes. The 2009 American Hospital Association Annual Survey Database provided hospital characteristics. ${ }^{16}$ The University of Michigan institutional review board for human subjects determined that this study did not require institutional review board regulation or approval.

\section{Study Population}

Appendix Figure S1 diagrams the application of adult patient and hospital exclusion criteria for constructing the analytical data set. Analyses were conducted specific to individuals with Medicare listed as the primary payer (including fee-for-service, managed care, and health maintenance organization) and an all-payer population because the HACI policy rapidly expanded to other payers. ${ }^{12,17}$ Hospitals not affected by the HACI, such as long-term care, rehabilitation, and psychiatric facilities and critical access, Veterans Affairs, and children's hospitals were excluded. Hospitals that did not have data available from 2007 and 2009 were also excluded.

\section{Pressure Ulcer Case Identification}

As detailed in Appendix Table S1, pressure ulcers were identified in administrative data according to International Classification of Diseases, Ninth Revision, Clinical Modification diagnoses ${ }^{11,14,18}$ specific to ulcer location (in 2007 and 2009) and severity according to stage (in 2009).
Assessing Statewide Rates of Present-on-Admission and Hospital-Acquired Pressure Ulcers in 2007 (Pre-Policy) and 2009 (Post-Policy)

Statewide rates of pressure ulcers in 2007 and 2009 were computed as the percentage of all adult discharges having at least one pressure ulcer diagnosis, categorized as present-on-admission versus hospital-acquired diagnoses.

\section{Assessing Hospital Payment Reductions in 2009 Due to HACI Payment Changes for Pressure Ulcers}

How often and by how much the HACI payment changes for pressure ulcers reduced hospital payment (in dollars) was determined using post-policy 2009 administrative data, MS-DRG software, and CMS hospital base payment files while accounting for the 2008 pressure ulcer diagnosis code changes, including pressure ulcer stage and status upon admission (Appendix S1, Appendix S2) and associated changes in qualification for outlier payment (as detailed in Appendix S3). In brief, according to the HACI, advanced-stage pressure ulcers (Stage III or IV) no longer can generate extra payment when they are hospitalacquired diagnoses, and neither can earlier-stage (Stage I and II) pressure ulcers, stage-not-specified or unstageable pressure ulcers generate extra payment as comorbidities whether hospital-acquired or present-on-admissoin diagnoses. Present-on-admission unstageable pressure ulcers cannot generate comorbidity payment by the $\mathrm{HACI}^{10,11,13}$ despite clinical definitions ${ }^{1}$ indicating unstageable pressure ulcers are clinically considered Stage III or IV.

\section{RESULTS}

Statewide Rates of Present-on-Admission and HospitalAcquired Pressure Ulcers in 2007 (Pre-Policy) and 2009 (Post-Policy)

There were 2,401,269 adult discharges from 311 California hospitals in 2007 and 2,490,488 discharges from the same hospitals in 2009 (Appendix Figure S1). Considering all stages and locations (Appendix Table S1), pressure ulcers were listed as present-on-admission diagnoses for $56,531(2.3 \%)$ discharges in 2007 and 74,684 (3.0\%) discharges in 2009. Hospital-acquired pressure ulcers were listed for 6,705 $(0.28 \%)$ discharges in 2007 and 6,654 $(0.27 \%)$ discharges in 2009 .

\section{Effect of Payment Changes for Pressure Ulcers on Hospital Payment}

Figure 1 outlines the number of discharges and payment changes that occurred as a direct consequence of the 2008 HACI changes in payment for pressure ulcers. According to pressure ulcer stage (available in 2009, Table 1), diagnoses of Stage III and IV hospital-acquired pressure ulcers occurred in 603 discharges $(0.02 \%)$; Stage I and II, unstageable, and stage-not-specified ulcers (including hospitalacquired and present-on-admission diagnoses) occurred in 60,244 discharges (2.4\%). Beginning with 78,114 hospitalizations in 2009 with at least one pressure ulcer diagnosis, 23,250 hospitalizations would have no payment change 


\section{8,114 California discharges in 2009 with at least one pressure ulcer diagnosis}

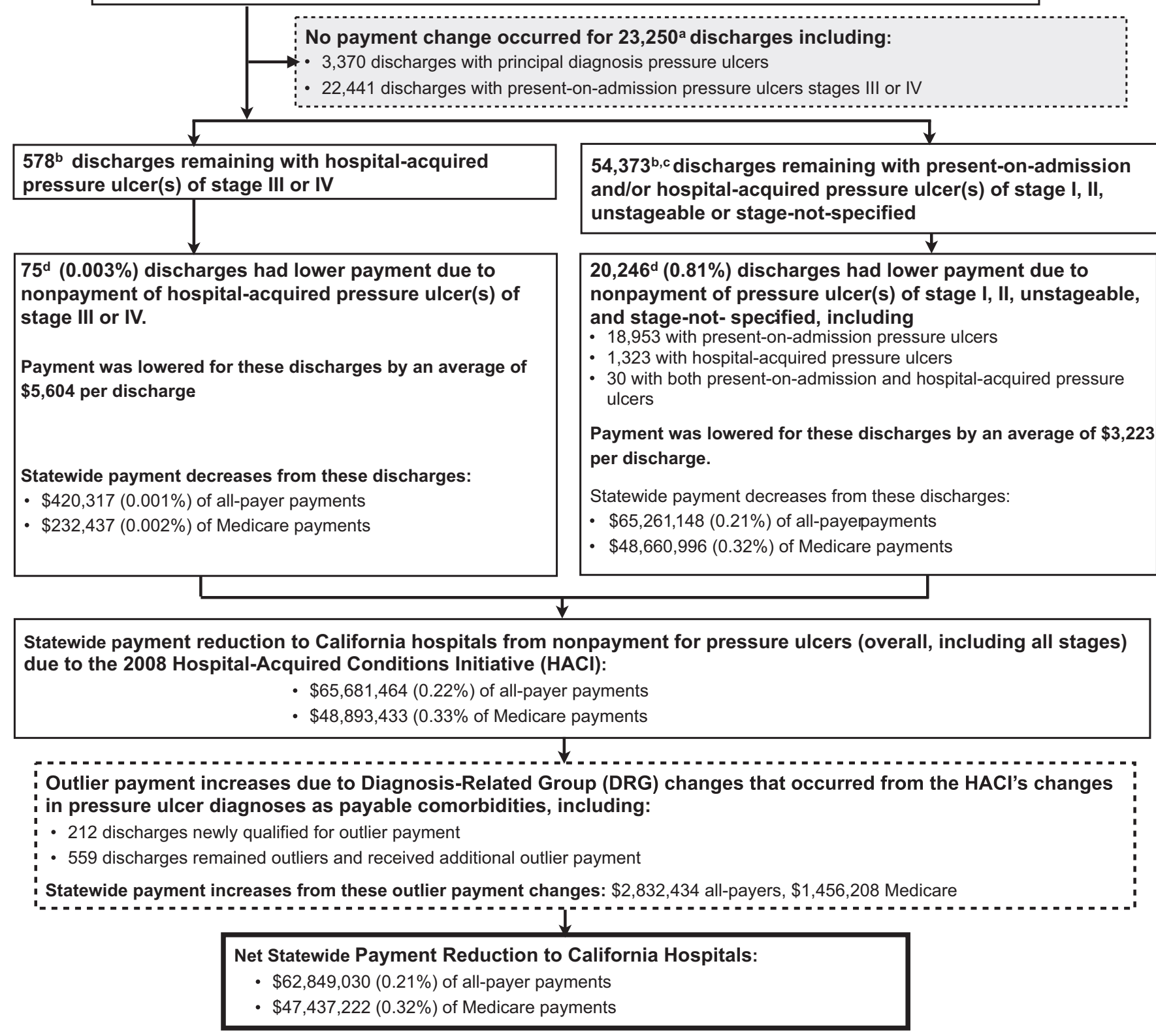

Figure 1. Flow diagram of payment changes for pressure ulcer diagnoses for California hospitals in 2009 due to 2008 changes in pressure ulcer payment in the Hospital-Acquired Conditions Initiative. ${ }^{\mathrm{a}} \mathrm{N}=2,561$ discharges had pressure ulcer listed as principal diagnosis and a present-on-admission Stage III or IV pressure ulcer. ${ }^{b} \mathrm{~N}=87$ discharges were eligible for both scenarios of payment reduction (had hospital-acquired Stage III or IV and another type of pressure ulcer). 'Stage I and II, unstageable, and stage-not-specified ulcers are no longer eligible for payment whether present-on-admission or hospital-acquired diagnoses. ${ }^{\mathrm{d}} \mathrm{N}=18$ discharges experienced a payment reduction under both scenarios.

because of having a principal diagnosis of pressure ulcer or having a present-on-admission Stage III or IV pressure ulcer. Nonpayment for hospital-acquired Stage III and IV pressure ulcers lowered hospital payment for $75(0.003 \%)$ hospitalizations. Nonpayment for Stage I and II, unstageable, and stage-not-specified pressure ulcers lowered hospital payment for $20,246(0.81 \%)$ hospitalizations. The remaining hospitalizations did not experience a reduction in payment because of the existence of other comorbidities that justified greater reimbursement (Appendix S2). Pressure ulcer payment changes moved 212 discharges to qualify for new outlier status and payment; 559 additional discharges maintained outlier status but received greater outlier payment because of the DRG change (Appendix S3).

The net dollar effect was next estimated considering pressure ulcer payment decreases and outlier payment increases using the hospital-specific base payment rates (Figure 1, bottom box). California hospitals experienced a total payment reduction of $\$ 62,849,030(0.21 \%)$ from all payers, including $\$ 47,437,222(0.32 \%)$ from Medicare. Nonpayment for hospital-acquired Stage III and IV pressure ulcers led to a net all-payer payment reduction of $\$ 310,444(0.5 \%$ of total reduction in all-payer payments, 
Table 1. Pressure Ulcers According to Stage for California Hospitals in 2009

\begin{tabular}{|c|c|c|c|}
\hline & $\begin{array}{l}\text { Present-on- } \\
\text { Admission } \\
\text { Pressure } \\
\text { Ulcers }^{a}\end{array}$ & $\begin{array}{l}\text { Hospital- } \\
\text { Acquired } \\
\text { Pressure } \\
\text { Ulcers }^{a}\end{array}$ & $\begin{array}{l}\text { Total Discharge } \\
\text { Records with a } \\
\text { Pressure Ulcer of } \\
\text { This Stage }\end{array}$ \\
\hline Stage & \multicolumn{3}{|c|}{$\begin{array}{c}\text { N (\% of Total Analytical Sample in } 2009 \text { with } \\
2,490,488 \text { Discharges) }\end{array}$} \\
\hline | & $13,080(0.53)$ & $1,374(0.06)$ & $14,454(0.58)$ \\
\hline II & 31,962 (1.28) & $3,271(0.13)$ & $35,233(1.41)$ \\
\hline III & $12,738(0.51)$ & $450(0.02)$ & $13,188(0.53)$ \\
\hline IV & $11,184(0.45)$ & $163(<0.01)$ & $11,347(0.46)$ \\
\hline $\begin{array}{l}\text { Stage-not- } \\
\text { specified }\end{array}$ & $9,381(0.38)$ & $711(0.03)$ & $10,092(0.41)$ \\
\hline Unstageable & 4,745 (0.19) & $235(<0.01)$ & $4,980(0.20)$ \\
\hline
\end{tabular}

Rows are not mutually exclusive because there could be multiple pressure ulcers per discharge.

${ }^{\mathrm{a}}$ Two hundred fifty-nine hospitalizations had both a present-on-admission and hospital-acquired pressure ulcer diagnosis listed as different stages.

Figure 2). California hospitals lost $\$ 5,687,255$ (9.0\% of total payment reductions for pressure ulcers, Figure 2) related to all other stages of hospital-acquired pressure ulcers and $\$ 56,851,331$ (90.5\% of reduced payments for pressure ulcers, Figure 2) for pressure ulcers described as present-on-admission Stage I and II, unstageable, and stage-not-specified ulcers. As Figure 2 emphasizes, the largest proportion of statewide payment reduction resulted from nonpayment $(\$ 62,538,586)$ for Stage I and II, unstageable, and stage-not-specified ulcers, which no longer count as payable comorbidities; this payment reduction was more than 200 times as great as for hospital-acquired Stage III and IV ulcers $(\$ 310,444)$.

For an average hospital, the payment reduction for hospital-acquired Stage III and IV pressure ulcers (including outlier payment changes) was $\$ 998$ from all payers $(0.001 \%$ of the hospital's total payments), including $\$ 641$ from Medicare $(0.001 \%$ of the hospital's Medicare payments). For an average hospital, payment reduction for all other stages of pressure ulcers (including outlier payment changes) was $\$ 201,089$ from all payers $(0.2 \%$ of the hos- pital's total payments), including $\$ 151,891$ from Medicare $(0.3 \%$ of the hospital's Medicare payments).

\section{DISCUSSION}

The total financial effect of the 2008 HACI payment changes for pressure ulcers was negligible. Within the small $(<0.4 \%)$ payment decrease that occurred, the largest proportion resulted from nonpayment for Stage I and II, unstageable, and stage-not-specified ulcers, the overwhelming majority $(90.5 \%)$ of which was due to nonpayment for present-on-admission ulcers - effectively a price cut unrelated to the care delivered. This payment change was more than 200 times as great as the reduction for hospitalacquired Stage III and IV ulcers-the pressure ulcers described in CMS information material ${ }^{9,10,14}$ summarizing the HACI for Medicare providers, and publicly reported from 2011 to 2013 on Medicare's Hospital Compare. ${ }^{19}$ Because the HACI policy's implementation includes price cuts unrelated to quality of care, hospitals are unable to preserve their payments by delivering higher-quality care. Removing many present-on-admission pressure ulcers as payable comorbidities also provided an unintentional disincentive for hospitals to avoid admitting patients with preexisting early ulcers, although many hospitals may be unaware of the removal of early pressure ulcers as payable comorbidities, as detailed only in the Federal Register. ${ }^{13}$

Hospital-acquired pressure ulcer rates (including all stages) remained low and relatively unchanged in administrative data in 2007 and 2009; pressure ulcers recorded as present-on-admission diagnoses increased $(2.3 \%$ in 2007 , $3.0 \%$ in 2009). The total financial effect of the 2008 payment changes was small for all-payer $(0.21 \%)$ and Medicare $(0.32 \%)$ statewide hospital payments.

Regarding limitations, this study involves one state's data for 2 years, although California has applied the present-on-admission variable since 1997 to identify hospitalacquired conditions, which became mandatory nationwide in October 2007; 2009 to 2011 trends in pressure ulcer diagnoses using the new stage-specific codes in administrative data demonstrated no significant changes (Appendix S4, Appendix Table S2). This study assesses the payment changes occurring as direct consequences of the HACI

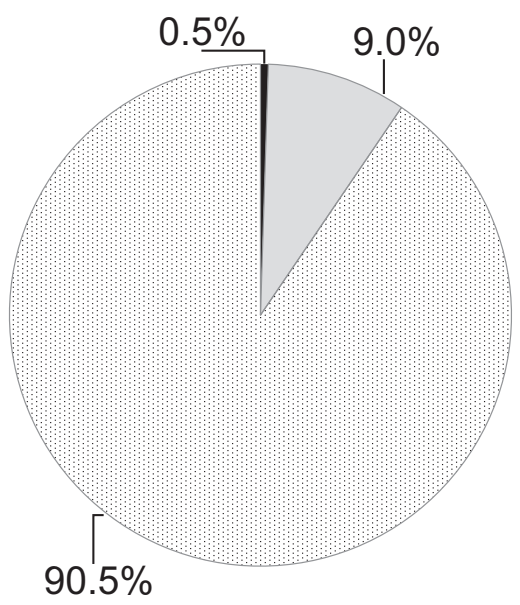

Stage III and IV hospital-acquired pressure ulcers

Stage I and II, unstageable, and stagenot-specified hospital-acquired pressure ulcers

Stage I and II, unstageable, and stagenot-specified present-on-admission pressure ulcers, including 30 discharges with both present-onadmission and hospital-acquired pressure ulcers

Figure 2. Percentage of total hospital payment reductions (according to pressure ulcer type) due to pressure ulcer payment changes by the 2008 Hospital-Acquired Conditions Initiative, California 2009. 
regarding pressure ulcers, not other conditions. Changes assessed using administrative data chosen for HACI implementation may not reflect actual changes in pressure ulcer rates experienced by patients, as supported by an analy$\mathrm{sis}^{20}$ demonstrating much lower pressure ulcer rates in administrative data than in surveillance pressure ulcer data.

Intended outcomes of the HACI included fewer hospital-acquired complications for patients and lower hospital payment for pressure ulcers. The intended decrease in hospital-acquired pressure ulcers was not seen in the administrative data selected for the HACI implementation in California from 2007 to 2009. Not surprisingly, the HACI decreased hospital payments attributed to pressure ulcer diagnoses, which removed all pressure ulcers except present-on-admission Stage III and IV ulcers from qualifying as payment-generating comorbidities, but payments decreased by only a tiny amount. It is unclear whether the increase in present-on-admission pressure ulcer rates (all stages) from $2.3 \%$ in 2007 to $3.0 \%$ in 2009 was related to the 2008 changes in pressure ulcer diagnosis codes or was an unintended consequence because hospitals have an overall incentive to document all present-on-admission conditions to avoid potential nonpayment or reporting as hospitalacquired conditions.

\section{CONCLUSION}

The total financial effect of the 2008 HACI payment changes for pressure ulcers was inconsequential, resulting in no significant financial penalty for hospitals and no significant savings for Medicare. Most payment decreases occurred by removal of comorbidity payments for presenton-admission pressure ulcers other than Stage III and IV. The removal of payment for hospital-acquired Stage III and IV ulcers by implementation of the HACI policy was $1 / 200$ th that of the removal of payment for other types of pressure ulcers that occurred in implementation of the HACI. It is likely that the much larger removal of payment for pressure ulcers other than hospital-acquired Stage III and IV ulcers will come as a surprise to most hospitals because the policy's description ${ }^{9,10,14}$ in press releases and on-line material prepared for Medicare providers describes payment removal only for hospital-acquired Stage III and IV ulcers. Although removal of payment for hospitalacquired Stage III and IV ulcers is consistent with trying to motivate hospitals to deliver higher value in quality of care purchased with healthcare dollars, the removal of comorbidity payment for present-on-admission pressure ulcers is a simple price cut without respect to value.

\section{ACKNOWLEDGMENTS}

The authors appreciate the insight and comments (uncompensated) provided by Gwendolyn Blackford, BS, about processes used and regulations followed by hospital coders while assigning diagnosis codes. They also thank Jack Hughes, MD, for his helpful suggestions and to Richard Averill, MS, MA, BS, and 3M Health Information Systems for making the MS Grouper Software available to assess changes in hospital payment. The authors thank Helen McGuirk, MPH, and Jason Mann, MSA, for assistance with references and manuscript editing. Presented at the 2013 Society of General Internal Medicine 35rd Annual Meeting, Denver, Colorado, and by poster presentation at the Academy Health 2013 Annual Research Meeting, Baltimore, Maryland.

Conflict of Interest: Dr. Meddings has reported receiving honoraria for lectures and teaching related to prevention and value-based purchasing policies involving catheter-associated urinary tract infection and hospitalacquired pressure ulcers.

The study was funded by grant 1R01HS018334 (PI: Dr. McMahon) from the Agency for Healthcare Research and Quality (AHRQ). Dr. Meddings's work was also funded by grant 1K08HS019767 (PI: Dr. Meddings) from AHRQ. Dr. Meddings was also a recipient of the 2009 to 2015 National Institutes of Health Clinical Loan Repayment Program.

Author Contributions: Dr. Meddings and Ms. Reichert had full access to all of the data in the study and take responsibility for the integrity of the data and the accuracy of the data analysis. Study concept and design: Meddings, Reichert, McMahon, Grazier. Acquisition of data: Meddings, Reichert. Analysis and/or interpretation of data: Meddings, Reichert, Grazier, Rogers. Drafting of the manuscript: Meddings, Reichert. Critical revision of the manuscript for important intellectual content: All authors. Statistical analysis: Reichert, Meddings. Obtaining funding: McMahon, Meddings. Study supervision: Meddings. The findings and conclusions in this report are those of the authors and do not necessarily represent those of the sponsor, the Agency for Healthcare Research and Quality.

Sponsor's Role: The AHRQ was not involved in study design or conduct; data collection, management, analysis, or interpretation; preparation, review, or approval of manuscript; or the decision to submit the manuscript for publication.

\section{REFERENCES}

1. National Pressure Ulcer Advisory Panel. NPUAP Pressure Ulcer Stages/ Categories [on-line]. Available at http://www.npuap.org/resources/educational-and-clinical-resources/npuap-pressure-ulcer-stagescategories/ Accessed October 21, 2014.

2. Beckrich K, Aronovitch SA. Hospital-acquired pressure ulcers: A comparison of costs in medical vs. surgical patients. Nurs Econ 1999;17:263-271.

3. Preventing Pressure Ulcers in Hospitals: A Toolkit for Improving Quality of Care (AHRQ Publication No. 11-0053-EF). Rockville, MD: Agency for Healthcare Research and Quality [on-line]. Available at http://www.ahrq.gov/research/ltc/pressureulcertoolkit/putoolkit.pdf Accessed March 5, 2014.

4. Pieper B, Langemo D, Cuddigan J. Pressure ulcer pain: A systematic literature review and National Pressure Ulcer Advisory Panel white paper. Ostomy Wound Manage 2009;55:16-31.

5. Lyder CH, Preson J, Grady JN et al. Quality of care for hospitalized Medicare patients at risk for pressure ulcers. Arch Intern Med 2001;161:15491554 .

6. Brem H, Maggi J, Nierman D et al. High cost of stage IV pressure ulcers. Am J Surg 2010;200:473-477.

7. Black J, Edsberg LE, Baharestani MM et al. Pressure ulcers: Avoidable or unavoidable? Results of the National Pressure Ulcer Advisory Panel Consensus Conference. Ostomy Wound Manage 2011;57:24-37.

8. The Patient Protection and Affordable Care Act, Section 3008: Payment adjustment for conditions acquired in hospitals. Pub L No. 111-148, 124 Stat 122 [on-line]. Available at http://www.gpo.gov/fdsys/pkg/PLAW111publ148/html/PLAW-111publ148.htm Accessed October 21, 2014.

9. U.S. Department of Health \& Human Services and the Centers for Medicare \& Medicaid Services, Hospital-Acquired Conditions (HAC) in Acute 
Inpatient Prospective Payment System (IPPS) Hospitals. Prepared by the Medicare Learning Network, Official CMS information for Medicare fee-for-service providers [on-line]. Available at http://www.cms.gov/ Outreach-and-Education/Medicare-Learning-Network-MLN/MLNProducts/ Downloads/wPOAFactSheet.pdf Accessed October 22, 2014.

10. Centers for Medicare \& Medicaid Services. Hospital-acquired conditions [online]. Available at http://www.cms.gov/Medicare/Medicare-Fee-for-ServicePayment/HospitalAcqCond/Hospital-Acquired_Conditions.html Accessed October 21, 2014.

11. Centers for Medicare and Medicaid Services, Department of Health and Human Services. Medicare program; changes to the hospital inpatient prospective payment systems and fiscal year 2009 rates. Fed Regist 2008;73:48434-49083.

12. Medicaid Program. Payment adjustment for provider-preventable conditions including health care-acquired conditions. Fed Regist 2011;76:3281632838 .

13. Centers for Medicare and Medicaid Services, Department of Health and Human Services. Medicare program; proposed changes to the hospital inpatient prospective payment systems and fiscal year 2009 rates. Fed Regist 2008;73:23547-23552.

14. Centers for Medicare and Medicaid Services. Fact sheet: incorporating selected National Quality Forum and Never Events into Medicare's list of hospital-acquired conditions. Press release: April 14, 2008 [on-line]. Available at http://www.cms.gov/Newsroom/MediaReleaseDatabase/Factsheets/2008-Fact-sheets-items/2008-04-142.html Accessed October 21, 2014.

15. Agency for Healthcare Research and Quality. Healthcare cost and utilization project overview and description of state inpatient databases [on-line]. Available at http://www.hcup-us.ahrq.gov/sidoverview.jsp Accessed March 3, 2014.

16. American Hospital Association. Data collection methods. Health Forum, LLC; 2012 [on-line]. Available at http://www.ahadataviewer.com/about/ data Accessed October 21, 2014.

17. Blue Cross and Blue Shield Announces System-Wide Payment Policy for "Never Events." Washington, DC: Blue Cross Blue Shield Association. Press release: February 26, 2010.

18. ICD-9-CM Official Guidelines for Coding and Reporting. Centers for Medicare and Medicaid Services (CMS) and the National Center for Health Statistics (NCHS). Effective October 1, 2008 [on-line]. Available at http://www. ama-assn.org/resources/doc/cpt/icd9cm_coding_guidelines_08_09_full.pdf Accessed March 5, 2014.
19. Centers for Medicare and Medicaid Services, U.S. Department of Health and Human Services. Medicare's Hospital Compare website measures for readmissions, complications and deaths, including hospital-acquired complications [on-line]. Available at http://www.medicare.gov/HospitalCompare/About/HOSInfo/RCD.aspx Accessed October 21, 2014.

20. Meddings J, Reichert H, Hofer T et al. Hospital report cards for hospitalacquired pressure ulcers: How good are the grades? Ann Intern Med 2013;159:505-513.

\section{SUPPORTING INFORMATION}

Additional Supporting Information may be found in the online version of this article:

Appendix Figure S1. Study flow diagram.

Appendix S1. Changes in pressure ulcer diagnosis ICD-9-CM codes related to the 2008 Hospital-Acquired Conditions Initiative.

Appendix S2. Identification of hospital discharges with reduced hospital payment due to the pressure ulcer payment changes in the 2008 Hospital-Acquired Conditions Initiative.

Appendix S3. Calculation of outlier payments.

Appendix S4. Trends in Pressure Ulcer Diagnosis Code use, 2009-2011.

Appendix Table S1. Pressure ulcer diagnosis ICD-9CM Codes.

Appendix S2. Percentage of Adult discharges in California hospitals with stage III and IV pressure ulcers, from 2009-2011.

Please note: Wiley-Blackwell is not responsible for the content, accuracy, errors, or functionality of any supporting materials supplied by the authors. Any queries (other than missing material) should be directed to the corresponding author for the article. 